\title{
Late time transition in the evolution of the Universe
}

\author{
Ekrem Aydiner ${ }^{1, *}$, Işıl Basaran-Oz ${ }^{1}$, Tekin Dereli ${ }^{2}$, and Mustafa Sarısaman ${ }^{1}$
}

\author{
${ }^{1}$ Department of Physics, Faculty of Science, İstanbul University, 34134, İstanbul, Turkey \\ ${ }^{2}$ Department of Physics, Faculty of Science, Koç University, 34450, İstanbul, Turkey \\ *ekrem.aydiner@istanbul.edu.tr
}

\begin{abstract}
The late time crossover from a power-law to an exponential expansion of the Universe evolution is the major problem in today's physical cosmology. Unless this critical transition problem is solved, it is not possible to reach a holistic theory of cosmology. In this study, we propose a simple model in the FLRW framework, where dark matter and dark energy interact through a potential. We analytically solve this model and obtain scale factor $a(t)$ from the presented model. Mainly, employing numerical solutions we show that the scale parameter has a hybrid form which includes power and exponential terms. The numerical results clearly show that there is a time crossover $t_{c}$ in the scale factor $a(t)$ curve, which indicates the transition from the power-law to the exponential expansion of the Universe. We fit these unscaled curves and obtain that scale factor behaves as $a(t) \propto t^{2 / 3}$ below $t \leq t_{c}$, and as $a(t) \propto \exp \left(H_{0} t\right)$ with $H_{0}=0.4$ and $H_{0}=0.3$ for the relatively weak and strong interactions above $t>t_{c}$, respectively. It is the first time that we explicitly obtain a hybrid scale factor incorporating the power and exponential terms as $a(t) \propto t^{2 / 3} e^{H_{0} t}$. We conclude that the presented model can solve the late time transition problem of the Universe based on dark matter and dark energy interaction. Additionally, we numerically obtain other kinematic parameters depending upon the scale factor. We discuss the limit behaviors of all relevant cosmological parameters. Our results are completely in good agreement with observational data. Finally, we state that this work makes essential steps towards solving a critical outstanding problem of the cosmology, and has a potential to creates a paradigm for future studies in this field.
\end{abstract}

\section{Introduction}

In 1998, the accelerated expansion of the Universe was conjectured by two groups based on observations of Type Ia Supernova $(\mathrm{SN} \mathrm{Ia})^{1,2}$. After this important observational discovery, the physical origin or mechanism of the accelerated expansion of the Universe became the most important challenge in modern cosmology. It was unexpected that the Universe was expanding with acceleration. We know from the time-line of the Universe evolution that after early time inflation ${ }^{3,4}$ the Universe passes through periods of radiation and matter-dominated eras, respectively. It is well-known that the evolution of the scale factor takes the functional form of $a(t) \propto t^{1 / 2}$ in the radiation-dominated era. Meanwhile, it is given as $a(t) \propto t^{2 / 3}$ in the matterdominated era in the FLRW metric. These parameters can be obtained by solving the Friedmann equations. It is noticed that the expansion of the Universe in both periods is represented by the power-laws. However, the supernova observations were surprisingly different and indicate that the Universe is expanding exponentially as $a(t) \propto \exp \left(H_{0} t\right)$. Thus, the physical origin of exponential expansion appears to be a big challenging problem of current physical cosmology. It is intriguing to see that people have tried to explain this period by introducing the cosmological constant $\Lambda$ or dark energy (DE) in the FLRW framework. This period is commonly called as dark-energy-dominated era. The physical origin of dark energy still remains to be a mystery. People suggested many interesting models in the literature trying to explain the origin of dark energy such as quintessence ${ }^{5}$, phantom ${ }^{6}$, -essence $^{7}$, tachyon ${ }^{8}$, Chaplygin gas ${ }^{9}$, holographic dark energy ${ }^{10}$. However, these models do not provide a satisfactory answer to the most major problem of the transition from a power-law to an exponential expansion. This time crossover is one of the important cosmology problems and is known as a late time transition. In fact, is the reason of this transition the existence of dark energy? Even if dark energy can be considered to be the cause of late time exponential expansion of the Universe, the existence of dark energy alone does not seem to be sufficient to explain this transition. Although numerous models have been proposed to explain this phenomenon, this transition still has not been explained consistently Refs. ${ }^{11-21}$. Without understanding this critical transition, it seems to be unlikely to proceed toward a comprehensive theory of cosmology.

Based on this motivation, in this study, we will focus on the solution of the late time transition of the Universe and propose a new model to solve this challenging problem of the cosmology. Before presenting the model, we would like to point out a few points. Firstly, we know that the scale factor is a quantity which characterizes the time-dependent expansion dynamics of the Universe. However, in the previous theoretical models, a generalized scale factor to characterize all phases of the Universe was not proposed or obtained. The other point is rather distinct from the first one where the key question is: Can we describe this critical transition as a phase transition or a catastrophic transition? If we view the problem in terms of the 
statistical mechanics of phase transitions, we can not say that it is a first or second-order phase transition. The turnover in the evolution process favors a catastrophic jump rather than a phase transition (Please see 22 for the catastrophic transitions). It is clear that the problem is more sophisticated, and requires an elaborate approach. Therefore, we may need new players to enlarge this discussion. Possible candidates are baryonic or non-baryonic dark matter (DM) and dark energy. Indeed, it was recently suggested that dark energy could be dynamic, evolving with time ${ }^{23-26}$. Because, we know that the dynamics of the realistic Universe is described by an EoS parameter which behaves differently at different epochs. Clearly, one can state that a single fluid with a constant can not give rise to a realistic cosmic history. Therefore, a realistic Universe model should be dominated by more ingredients which can be defined by different EoS parameters ${ }^{27}$. In such a model, the components interact and dominate the evolution of the Universe ${ }^{28}$.

Indeed, it is shown in the literature that the interacting models have potential to solve many problems of the cosmology. For example, many interacting models have been used to solve the singularity and cosmic coincidence problems ${ }^{29-52}$. More recently, a different interaction model has been introduced by Aydiner in Ref. ${ }^{28}$. In his study, it has been shown that the interaction between matter, dark matter and dark energy has lead to the chaotic evolution of the Universe. It was seen that this model combined the big bang model and the oscillatory Universe models, as well as had the potential to solve many fundamental problems of cosmology such as singularity, the future of the Universe, the formation of the galaxies and largescale organization of the Universe. However, the late time transition was not specifically discussed by Aydiner in Ref. ${ }^{28}$ and others. These studies provide a possible solution to the late time transition of the Universe based on interactions between dark energy and dark matter. Therefore, our aim in this study is to discuss the late time transition of the Universe based on the interaction of dark matter and dark energy. Here, for simplicity, we define these components as the two different scalar fields in the theoretical framework of the FLRW metric. We consider the interaction between them and generalize the model based on the motivation in Ref. ${ }^{53}$.

To discuss the time crossover between power-law and exponential expansion of the Universe evolution, we set a Lagrangian based on the FLRW metric with two scalar fields interacting by a potential term. We introduce a suitable and exactly solvable potential from an interacting model which includes higher-order terms having a potential of catastrophic behaviour in dynamical evolution. We analytically solve this model and obtain the scale factor. By using the presented model we show that it is possible to explain the mechanism of this time crossover in the late-time evolution of the Universe. Additionally, we obtain and discuss other cosmological parameters such as Hubble $H(t)$, deceleration $q(t)$, the equation of states (EoS) $w$ and jerk parameters $j(t)$ for this model.

In summary, we explicitly obtain a hybrid scale factor incorporating the power and exponential terms as $a(t) \propto t^{2 / 3} e^{H_{0} t}$. We show that the late time transition of the Universe can be explained based on dark matter and dark energy interaction.

\section{Interaction Between DM and DE}

In this study, we propose that DM and DE can be represented by two different scalar fields for instance $\phi$ and $\sigma$, and they interacts with a potential. In this case, the action of minimally coupled scalar gravity for two-scalar fields is described by

$$
S=\int d^{4} x \sqrt{-g}\left[\frac{R}{2 \kappa}+\frac{1}{2}\left(\partial_{\mu} \phi \partial^{\mu} \phi+\partial_{\mu} \sigma \partial^{\mu} \sigma\right)-V(\phi, \sigma)\right]
$$

where $\phi, \sigma: \mathbb{R}^{4} \rightarrow \mathbb{R}$ are scalar-valued $C^{\infty}$ fields, $V$ is the potential expressed as a function of the scalar fields, $R$ is the Ricci scalar, and $\kappa=8 \pi G / c^{4}$ is a constant that we use the geometric unit system, i.e. $\kappa=1$. Scalar fields are defined on a manifold with metric $\gamma_{a b}(\phi, \sigma)$ and action is invariant under the symmetries of the scalar fields.

Consider the FLRW metric expressing a homogeneous isotropic space-time metric given by

$$
d s^{2}=h_{\mu v} d x^{\mu} d x^{v}
$$

with the metric $h_{\mu v}=\operatorname{diag}\left(-1, a(t) I_{3}\right), I_{3}=\operatorname{diag}(1,1,1)$ is $3 \times 3$ identity matrix, $a(t): \mathbb{R} \rightarrow \mathbb{R}$ is a differentiable function which is known as time-dependent scale factor. The Ricci scalar equipped with this space-time (2) is specified by

$$
R=6\left(\frac{\ddot{a}}{a}+\frac{\dot{a}^{2}}{a^{2}}\right)
$$

where the dot denotes the derivative with respect to time. Now, the point-like Lagrangian for DM and DE interaction can be written as follow

$$
\mathscr{L}=-3 a \dot{a}^{2}+\frac{a^{3}}{2}\left(\dot{\phi}^{2}+\dot{\sigma}^{2}\right)-a^{3} V(\phi, \sigma) .
$$

At this point, we can obtain analytical solution using the Lagrangian (4). 


\section{Analytical Results}

One can easily reveal the set of equations of motion by means of the dynamical variables $\{a, \phi, \sigma\}$ for the Lagrangian (4). These are obtained as

$$
\begin{aligned}
2 \frac{\ddot{a}}{a}+\frac{\dot{a}^{2}}{a^{2}}+\frac{1}{2}\left(\dot{\phi}^{2}+\dot{\sigma}^{2}\right)-V(\phi, \sigma) & =0, \\
\ddot{\phi}+3 \frac{\dot{a}}{a} \dot{\phi}+\frac{\partial V(\phi, \sigma)}{\partial \phi} & =0, \\
\ddot{\sigma}+3 \frac{\dot{a}}{a} \dot{\sigma}+\frac{\partial V(\phi, \sigma)}{\partial \sigma} & =0 .
\end{aligned}
$$

Notice that once we impose the zero energy condition, the remaining equation necessary for this theory is obtained as follows

$$
-3 \frac{\dot{a}^{2}}{a^{2}}+\frac{1}{2}\left(\dot{\phi}^{2}+\dot{\sigma}^{2}\right)+V(\phi, \sigma)=0 .
$$

To solve the equations of motion in Eqs.(5) and (6) we need to determine an appropriate potential. In our study, we focus our attention on a specific potential characterized by

$$
2 \alpha^{2}\left(X_{1}^{2}-X_{2}^{2}+Y_{1}^{2}-Y_{2}^{2}\right) V(\phi, \sigma)=A_{1} X_{1}^{2}+A_{2} X_{2}^{2}+B_{1} Y_{1}^{2}+B_{2} Y_{2}^{2}+2 k_{1} X_{1} Y_{2}-2 k_{2} X_{2} Y_{1} .
$$

Based on our experiences we know that this potential linearizes the field equations to get the precise solutions. However, in order to see that this potential gives rise to the physically meaningful and stable solutions, we have to check the potential surface. Therefore, inspired by the mechanical analogy, we introduce the following transformations

$$
\begin{aligned}
X_{1} & =a^{3 / 2} \cosh (\alpha \phi), \\
X_{2} & =a^{3 / 2} \sinh (\alpha \phi), \\
Y_{1} & =a^{3 / 2} \cosh (\alpha \sigma), \\
Y_{2} & =a^{3 / 2} \sinh (\alpha \sigma),
\end{aligned}
$$

where $\phi, \sigma \in[-\infty, \infty]$ and $a: \mathbb{R} \rightarrow \mathbb{R}^{+}$. By using these transformations, we can write the potential $V$ which explicitly depends on the scalar fields $\phi$ and $\sigma$ as

$$
\begin{aligned}
V(\phi, \sigma) & =\frac{1}{2 \alpha^{2}}\left[A_{1} \cosh ^{2}(\alpha \phi)+A_{2} \sinh ^{2}(\alpha \phi)+B_{1} \cosh ^{2}(\alpha \sigma)+B_{2} \sinh ^{2}(\alpha \sigma)\right. \\
& \left.+k_{1} \cosh (\alpha \phi) \sinh (\alpha \sigma)-k_{2} \cosh (\alpha \sigma) \sinh (\alpha \phi)\right] .
\end{aligned}
$$

For the sake of simplicity, we set $k_{1}=-k_{2}=k$. Then, the potential becomes

$$
V(\phi, \sigma)=\frac{1}{2 \alpha^{2}}\left[\delta_{1}+\delta_{2} \sinh ^{2}(\alpha \phi)+\delta_{3} \sinh ^{2}(\alpha \sigma)+2 k \sinh [\alpha(\sigma-\phi)]\right]
$$

where $\delta_{1}=\left(A_{1}+B_{1}\right), \delta_{2}=\left(A_{1}+A_{2}\right), \delta_{3}=\left(B_{1}+B_{2}\right)$ and $\alpha^{2}=3 / 4$. Furthermore, the potential $V(\phi, \sigma)$ should have natural identifications for small $\phi$ and $\sigma$. Therefore, we realize that the coefficients of $\phi^{2} / 2$ and $\sigma^{2} / 2$ terms can be identified by the positive-valued mass terms $m_{\phi}^{2}$ and $m_{\sigma}^{2}$ respectively, and $V(0,0)$ by the cosmological constant $\Lambda$. Potential can be Taylor expanded up to the order of fifth terms as follows

$$
\begin{aligned}
V(\phi, \sigma) & =\frac{1}{2 \alpha^{2}}\left(A_{1}+B_{1}\right)+\frac{k}{\alpha}(\sigma-\phi)+\frac{1}{2}\left(A_{1}+A_{2}\right) \phi^{2}+\frac{1}{2}\left(B_{1}+B_{2}\right) \sigma^{2}+\frac{k \alpha}{2}\left(\phi^{2} \sigma-\sigma^{2} \phi\right) \\
& +\frac{k \alpha}{6}\left(\sigma^{3}-\phi^{3}\right)+\frac{k \alpha^{3}}{12}\left(\sigma^{3} \phi^{2}-\sigma^{2} \phi^{3}\right)+\mathscr{O}_{6}(\phi, \sigma),
\end{aligned}
$$

where $\mathscr{O}_{6}$ denotes the sixth and higher-order terms. We realize that this potential involves the associated potential terms in the catastrophic theory for small field variables.

In view of these results, the potential surface corresponding to $V$ governed by the field variables $\phi$ and $\sigma$ is displayed in Fig. 1. It is obvious that the potential surface has a global minimum in the limit of $k \rightarrow 0$ and $\nabla V(0,0) \rightarrow 0$. This minima guarantees that the solutions in Eq. (12) are stable. 


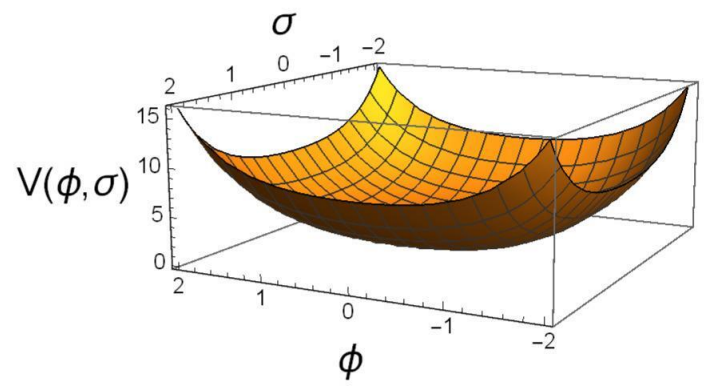

Figure 1. The potential surface of $V$ as a function of field variables $\phi$ and $\sigma$. We set values as $A_{1}=B_{2}=1.005$, $A_{2}=B_{1}=2.005, k=0.005$.

The stable solutions arise around the minimal potential. These stable solutions also give rise to the stable cosmological solutions. Therefore, we see that in the limit of $k \rightarrow 0$, we get $\nabla V(0,0) \rightarrow 0$ for the appropriate parameter values. Based on this idea, the cosmological constant and the mass parameters of the scalar fields are obtained respectively as,

$$
\begin{aligned}
\Lambda & :=V(0,0)=\frac{2}{3}\left(A_{1}+B_{1}\right), \\
m_{\phi}^{2} & :=\partial_{\phi}^{2} V(0,0)=\left(A_{1}+A_{2}\right), \\
m_{\sigma}^{2} & :=\partial_{\sigma}^{2} V(0,0)=\left(B_{1}+B_{2}\right) .
\end{aligned}
$$

Using the expression in (7) and the transformations in (8), new Lagrangian can be written as

$$
\mathscr{L}=\dot{X}_{1}^{2}-\dot{X}_{2}^{2}+\dot{Y}_{1}^{2}-\dot{Y}_{2}^{2}+A_{1} X_{1}^{2}+A_{2} X_{2}^{2}+B_{1} Y_{1}^{2}+B_{2} Y_{2}^{2}+2 k_{1} X_{1} Y_{2}-2 k_{2} X_{2} Y_{1} .
$$

Thus, by linearization, instead of nonlinear field equations in (5), four linear field equations are obtained as follows

$$
\begin{aligned}
\ddot{X}_{1} & =A_{1} X_{1}+k_{1} Y_{2}, \\
\ddot{X}_{2} & =-A_{2} X_{2}+k_{2} Y_{1}, \\
\ddot{Y}_{1} & =B_{1} Y_{1}-k_{2} X_{2}, \\
\ddot{Y}_{2} & =-B_{2} Y_{2}-k_{1} X_{1} .
\end{aligned}
$$

These equations can be expressed in a more compact form with the identification

$$
\xi=\left(\begin{array}{c}
X_{1} \\
X_{2} \\
Y_{1} \\
Y_{2}
\end{array}\right)
$$

such that (14) amounts to the following equation

$$
\ddot{\xi}=\mathbb{M} \xi \text {, }
$$

where $\mathbb{M}$ is the matrix obtained from the coefficients of field equations in (14). To find an appropriate solution of the Eq. (16), we first find out the eigenvalues and eigenvectors which are obtained by the characteristic equation $\operatorname{det}(\mathbb{M}-\lambda \mathbb{I})=0$. Thus, corresponding eigenvalues are attained as follows

$$
\begin{aligned}
& \lambda_{1,2}=\frac{1}{2}\left[A_{1}-B_{2} \pm \sqrt{\left(A_{1}+B_{2}\right)^{2}-4 k_{1}^{2}}\right], \\
& \lambda_{3,4}=\frac{1}{2}\left[-A_{2}+B_{1} \pm \sqrt{\left(A_{2}+B_{1}\right)^{2}-4 k_{2}^{2}}\right],
\end{aligned}
$$

where we denote the distinct eigenvalues by means of " \pm " sign elements. The corresponding eigenvectors can be obtained 
accordingly

$$
\begin{aligned}
& S_{1}\left(\lambda_{i}\right)=B_{2}+\sum_{i=1}^{4} \lambda_{i}-k_{1}, \\
& S_{2}\left(\lambda_{i}\right)=B_{1}-\sum_{i=1}^{4} \lambda_{i}+k_{2}, \\
& S_{3}\left(\lambda_{i}\right)=A_{2}+\sum_{i=1}^{4} \lambda_{i}+k_{2}, \\
& S_{4}\left(\lambda_{i}\right)=A_{1}-\sum_{i=1}^{4} \lambda_{i}-k_{1} .
\end{aligned}
$$

As a result, eigenvalues in Eq. (17) and eigenvectors in Eq. (18) give rise to the exact solutions of the field equations, which take the following form in components

$$
\xi_{i}=\sum_{i, j} S_{i}\left(\lambda_{j}\right)\left(\alpha_{j}+\beta_{j}\right)
$$

where $\alpha_{j}=m_{j} e^{\sqrt{\lambda_{j} t}}, \beta_{j}=n_{j} e^{-\sqrt{\lambda_{j} t}}, m_{j}$ and $n_{j}$ are constants and $i, j=1,2,3,4$.

In view of these findings, we now return to our main discussion to find the solutions of cosmological quantities. At this point, we can give solutions to cosmological quantities for the Lagrangian in Eq. (4) with two scalar fields. In fact, the basic cosmological parameter is the scale factor $a(t)$ that is completely independent of position or direction and tells us how the expansion or contraction of the Universe depends on the cosmic time. We can present the scalar factor $a(t)$ in terms of the new coordinate variables as

$$
a(t)=\left[\frac{1}{2}\left(\xi_{1}^{2}-\xi_{2}^{2}+\xi_{3}^{2}-\xi_{4}^{2}\right)\right]^{\frac{1}{3}} .
$$

On the other hand, the scalar fields $\phi(t)$ and $\sigma(t)$ that appear in the Lagrangian which represent dark matter and dark energy, respectively, are given in the form:

$$
\phi(t)=\frac{1}{\alpha} \tanh ^{-1}\left(\frac{\xi_{2}}{\xi_{1}}\right), \quad \sigma(t)=\frac{1}{\alpha} \tanh ^{-1}\left(\frac{\xi_{4}}{\xi_{3}}\right) .
$$

Other cosmological parameters such as Hubble $H$, deceleration $q$ and jerk $j$ parameters can be expressed in terms of $a, \dot{a}, \ddot{a}$ and $\dddot{a}$ as follows

$$
H:=\frac{\dot{a}}{a}, \quad q:=-\frac{\ddot{a}}{a H^{2}}, \quad j:=\frac{\dddot{a}}{a H^{3}} .
$$

These parameters can be determined from Taylor's expansion of the scale factor $a$ around $t=0$. Here, by definition, the Hubble parameter $H$ tells us the cosmic time-dependent expansion rate of the Universe, the Deceleration parameter $q$ tells us the change in the expansion rate of the Universe and the Jerk parameter $j$ tells us the change in the acceleration or deceleration of the Universe. Additionally, the effective equation of state (EoS) parameter is given in terms of the effective pressure $p_{e f f}$ and effective density $\rho_{e f f}$ as follows

$$
w_{e f f}=\frac{p_{e f f}}{\rho_{\text {eff }}},
$$

where the pressure is given by the expression $p_{e f f}=\frac{1}{2} \dot{\phi}^{2}+\frac{1}{2} \dot{\sigma}^{2}-V(\phi, \sigma)$, whereas the density is provided by $\rho_{\text {eff }}=$ $\frac{1}{2} \dot{\phi}^{2}+\frac{1}{2} \dot{\sigma}^{2}+V(\phi, \sigma)$.

\section{Numerical Results}

\section{The Scale Factor}

We solve field equations in Eq. (14) and analytically obtain the scale factor $a(t)$ in Eq. (20). Now, we give the numerical result of the scale factor in Fig. 2 for relatively weak and relatively strong interactions. In this numerical solutions, we set parameters arbitrarily as $A_{1}=B_{2}=1.005, A_{2}=B_{1}=2.005, k=0.005$ for the red circle line; $A_{1}=B_{2}=3.05, A_{2}=B_{1}=3.15, k=-2.95$ 
for the green star line, where here and in what follows, these parameters are selected such that minimal and stable potential together with minimal/maximal interactions for small/large $k$ values are guaranteed.

As it can be seen from Fig. 2 that scale factor and cosmic time represent the only parametric solution, both of which are unnormalized and unscaled to real cosmic data. In Fig. 2(a), the time dependent behavior of the scale factor is given. However, the scale factor is provided by log-log and semi-log scale in Fig. 2(b) and (c), respectively. The scale factor appears to increase exponentially or polynomially in Fig. 2(a). However, the real character of the time dependent behavior of the scale factor is displayed in Figs. 2(b) and (c). It can be observed from the log-log plot in Fig. 2(b) that the scale factor increases by a power-law up to a crossover time $t_{c}$ point. On the other hand, above this critical point, it increases exponentially as seen in Fig. 2(c). This crossover point indicates the transition from power-law expansion to the exponentially expanding era of the Universe. This extraordinarily important and surprising result solves the late-time transition problem which is one of the most important problems of cosmology. Furthermore, we find an even more surprising result in Fig. 2(b). The slope of the curve is approximately $2 / 3$. This unexpected result emerges another surprising result.
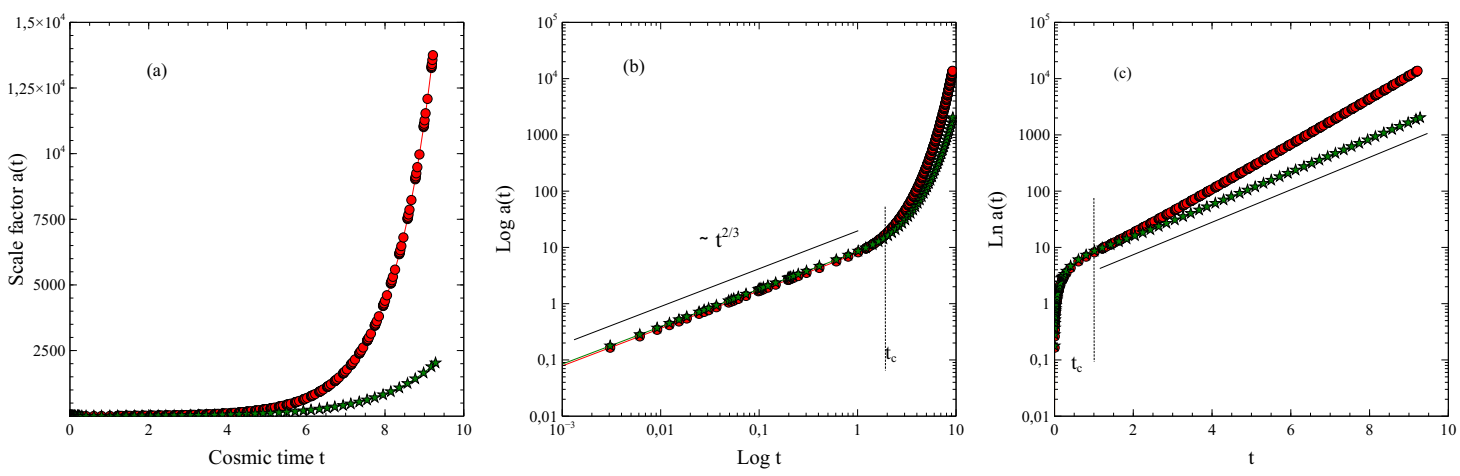

Figure 2. In (a), the scale factor $a(t)$ with respect to the cosmic time is given. Here we set the parameter values $A_{1}=B_{2}=1.005, A_{2}=B_{1}=2.005$ and $k=0.005$ for the red circle line; $A_{1}=B_{2}=3.05, A_{2}=B_{1}=3.15$ and $k=-2.95$ for the green star line. In (b), scale factor in Log-Log scale is given. In (c), scale factor $a(t)$ in semi-log plot is displayed. Here $t_{c}$ value is given by Eq. (25).

Our numerical results clearly show that the transition in the scale factor $a(t)$ can be represented by

$$
a(t) \propto \begin{cases}t^{2 / 3} & \text { for } t \leq t_{c}, \\ e^{H_{0} t} & \text { for } t>t_{c},\end{cases}
$$

where $H_{0}$ is the Hubble parameter. In our model, we find $H_{0} \simeq 0.4$ from the slope of the curve in Fig. 2(c). Here $t_{c}$ value can be found by the condition $t_{c}^{2 / 3}=e^{H_{0} t_{c}}$. Thus, $t_{c}$ is found by the following equation

$$
\frac{\ln t_{c}}{t_{c}}=\frac{3 H_{0}}{2}
$$

Therefore $t_{c}$ is computed to be $t_{c}=1.117$. The scale factor in Eq. (24) can be written in a single formula as

$$
a(t) \propto t^{2 / 3} e^{H_{0} t}
$$

We obtain this scale factor from our numerical result. For $t \leq t_{c}$, the first term $t^{2 / 3}$ dominates the scale factor a(t), however, for $t \leq t_{c}$, the exponential term $e^{H_{0} t}$ dominates the scale factor $a(t)$ in Eq. (26). Now, we can incorporate the result and our model as

$$
a(t)=\left[\frac{1}{2}\left(\xi_{1}^{2}-\xi_{2}^{2}+\xi_{1}^{2}-\xi_{2}^{2}\right)\right]^{\frac{1}{3}} \propto t^{2 / 3} e^{H_{0} t} .
$$

The first time, we precisely obtain a hybrid scale factor by using an interacting model. Suggested scale factor exhibits the characteristic feature of the expected crossover we seek for. As can be noticed, there will be combined effect of both factors presented in Eq. (24). That is the origin of this judicious choice of scale factor. In practice, there will be a range of this crossover that determines the domain of this hybrid scale factor. Here we note that choosing different parameter values does not change the character of the solution in Eq. (26). However, parameter values change, particularly, the slope of Fig. 2(c) and the position of the crossover time in the time axis. In the numerical procedure, we used arbitrary parameter values and obtained 
unscaled results. We know that the dark-energy-dominated era began after the matter-dominated era when the Universe was about 9.8 billion years old. On the other hand, the current value in the late time inflation phase is about $H_{0}=70.88 \mathrm{~km}$ $\mathrm{s}^{-1} \mathrm{Mpc}^{-1}$. Therefore, to obtain more correct $t_{c}$ and $H_{0}$ values from the model, the parameters of the model have to be fitted to the cosmological data, for instance BAO and SN Ia data.

According to our findings, we show that we can explain the late time crossover from power-law to exponential expansion of the Universe by using the FLRW model including DM and DE interactions. Furthermore, we explicitly obtain a real scale factor involving power and exponential terms in a single formula from the model. We report these results for the first time by using a model-dependent study.

\section{Other Kinematic Parameters}

In this section, we numerically obtain the Hubble parameter $H(t)$, the deceleration parameter $q(t)$, The jerk parameter $j(t)$ and the EoS parameter for relatively weak and relatively strong interactions. All of these parameters can be obtained from the scale factor $a(t)$ we obtained. In these numerical solutions, we set parameters arbitrarily as $A_{1}=B_{2}=1.005, A_{2}=B_{1}=2.005$ and $k=0.005$ for the red circle line, $A_{1}=B_{2}=3.05, A_{2}=B_{1}=3.15$ and $k=-2.95$ for the green star line in all figures below. Besides, the scale factor $a(t)$ and the other parameters are also not normalized and scaled to the observational cosmic data in the figure. We note that in this section we fit the curves to show the only time dependent characters of the quantities.

\section{The Hubble parameter $H(t)$}

The Hubble parameter is given by the ratio of the rate of change of the scale factor to the current value of the scale factor $a(t)$, which reflects the characteristic rate of the expansion of the Universe. The Hubble parameter can be obtained by using observational data, which depends on the red-shift. It takes different values for the radiation-dominated era, the matter-dominated era and late time inflation. In our case, Hubble parameter is obtained from the model. The time dependence of the Hubble parameter for the present model is provided in Fig. 3. The time-dependent behavior of the Hubble parameter is displayed in Fig. 3(a). However, the Hubble parameter is given by log-log and semi-log scales in Figs. 3(b) and (c), respectively.

It is noticed that the Hubble parameter has an anomaly depending on the scale factor $a(t)$. It starts from a maximum value and rapidly drops to a minimum value, and then reaches up to a maximum value with time. This minima corresponds to the critical transition time $t_{c}$ observed in scale factor behavior. Clearly, we expect the dramatic change of the Hubble parameter in the case of the phase-like catastrophic transition from matter dominate era to dark energy dominate era. However, this minima additionally emphasizes that before the catastrophic transition, there occurs a short deceleration in the expansion of the Universe. This is a very interesting point from which its physical meaning and mechanism can be discussed profoundly. In order to see some more details of the time evolution of the Hubble parameter, one can analyze the Figs. 3(b) and (c) further. In Fig. 3(b), it is seen that the Hubble parameter decreases as $H(t) \propto t^{-\alpha}$ up to the critical time point $t_{c}$. On the other hand, above $t_{c}$, it increases exponentially as $H(t) \propto e^{\alpha^{\prime} t}$ and it reaches up to a constant value with time for relatively weak and relatively strong interactions, where $\alpha$, and $\alpha^{\prime}$ denote arbitrary constant parameters.
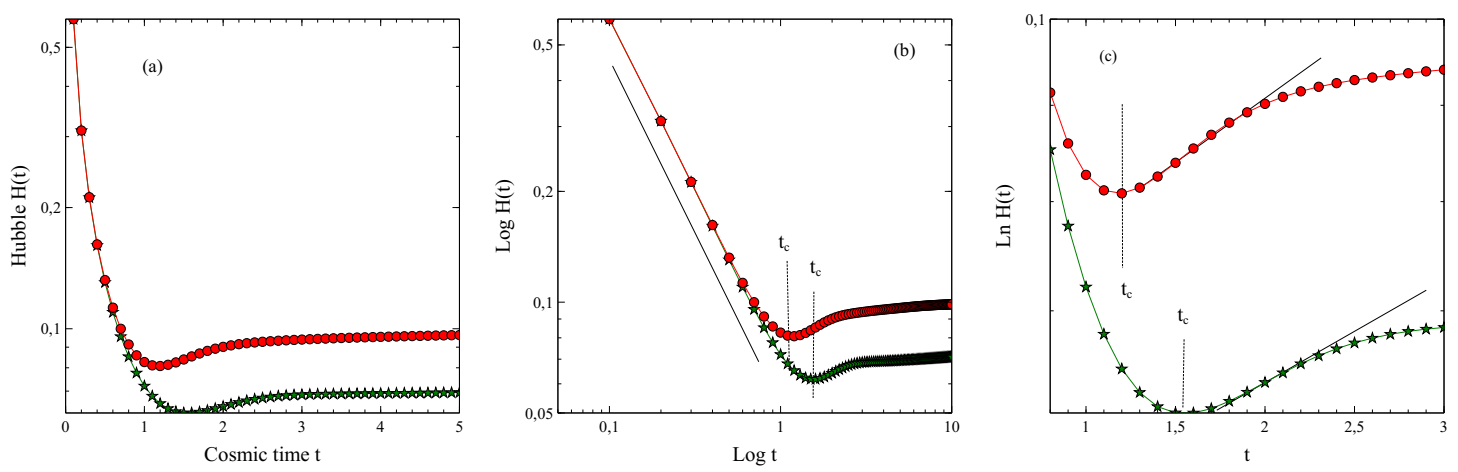

Figure 3. In (a), the Hubble parameter $H(t)$ with respect to cosmic time is given. Here we set the parameter values $A_{1}=B_{2}=1.005, A_{2}=B_{1}=2.005$ and $k=0.005$ for the red circle line; $A_{1}=B_{2}=3.05, A_{2}=B_{1}=3.15$ and $k=-2.95$ for the green star line. In (b), Hubble parameter $H(t)$ in Log-Log scale is displayed. In (c), Hubble parameter $H(t)$ in semi-log plot is shown.

\section{The deceleration parameter $q(t)$}

The deceleration parameter $q$ in cosmology is a dimensionless measure of the cosmic acceleration of the expansion of space in a FLRW Universe. In general, $q$ takes a negative sign and varies with the cosmic time, except in a few special cosmological 
models. Except in the speculative case of phantom energy, all postulated forms of mass-energy yield a deceleration parameter $q \geq-1$. On the other hand, for any non-phantom Universe, there must be a decreasing Hubble parameter, except in the case of the distant future of a $\Lambda \mathrm{CDM}$ model, where $q$ goes to -1 from above and the Hubble parameter gets asymptote to a constant value of $H_{0} \simeq \sqrt{\Lambda / 3}$. In our case, the deceleration parameter is obtained from the model itself. The time dependence of the
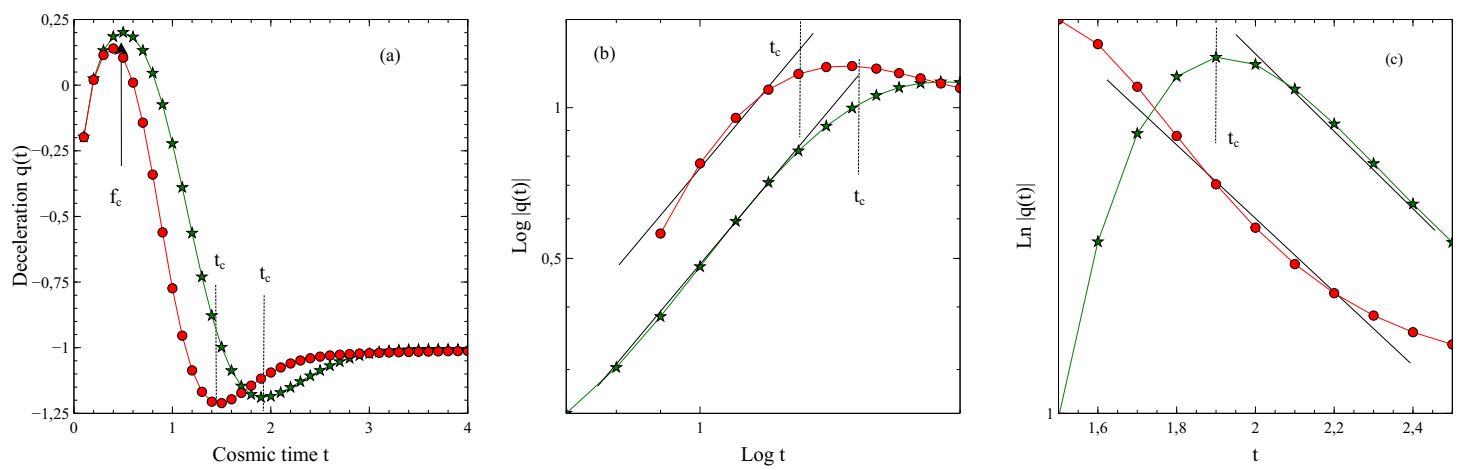

Figure 4. The deceleration parameter $q(t)$ with respect to cosmic time is displayed. Here we set the parameter values $A_{1}=B_{2}=1.005, A_{2}=B_{1}=2.005$ and $k=0.005$ for the red circle line; $A_{1}=B_{2}=3.05, A_{2}=B_{1}=3.15$ and $k=-2.95$ for the green star line. In (b), deceleration parameter $q(t)$ in $\log -\log$ scale is shown. In (c), deceleration parameter $q(t)$ in semi-log plot is provided.

deceleration parameter for the present model is shown in Fig. 4. The time dependent behavior of the deceleration parameter is indicated in Fig. 4(a). However, the deceleration parameter is given by log-log and semi-log scale in Figs. 4(b) and (c), respectively. We note that $\log -\log$ and semi-log figures are plotted for the absolute value of the deceleration parameter after first peaks $f_{c}$ to yield the slope of the curves. Therefore, in Figs. 4(b) and (c), curves occur inversely.

As can be clearly seen that the sign of the phase-like transition also appears at the critical crossover time values $t_{c}$ in Fig. 4(a). The deceleration parameter for the early time takes a positive value and rapidly drops to a minimum value at located $t_{c}$ as seen from Fig. 4(a). In order to see some details of the time evolution of the deceleration parameter, one can see the Figs. 4(b) and (c). In Fig. 4(b), it is seen that the Hubble parameter decreases as $q(t) \propto t^{-\beta}$ up to a critical time point $t_{c}$. On the other hand, after $t_{c}$, it increases exponentially as $H(t) \propto e^{\beta^{\prime} t}$ as seen in Fig. 4(c) and it reaches up to a constant value -1 with time for relatively weak and relatively strong interactions where $\beta$, and $\beta^{\prime}$ denote arbitrary constant parameters.

The jerk parameter $j(t)$

In cosmology, the jerk parameter $j(t)$ corresponds to the acceleration changes of expansion with respect to time. It is a very useful parameter to reveal the hidden transitions between phases of different cosmic accelerations. This parameter is defined as the dimensionless third derivative of the scale factor with respect to cosmic time. To confirm the presence of such a jump in the evolution of the expansion of the Universe, we carry out the presence of the phase-like catastrophic transition for our non-linear interacting two scalar fields model.
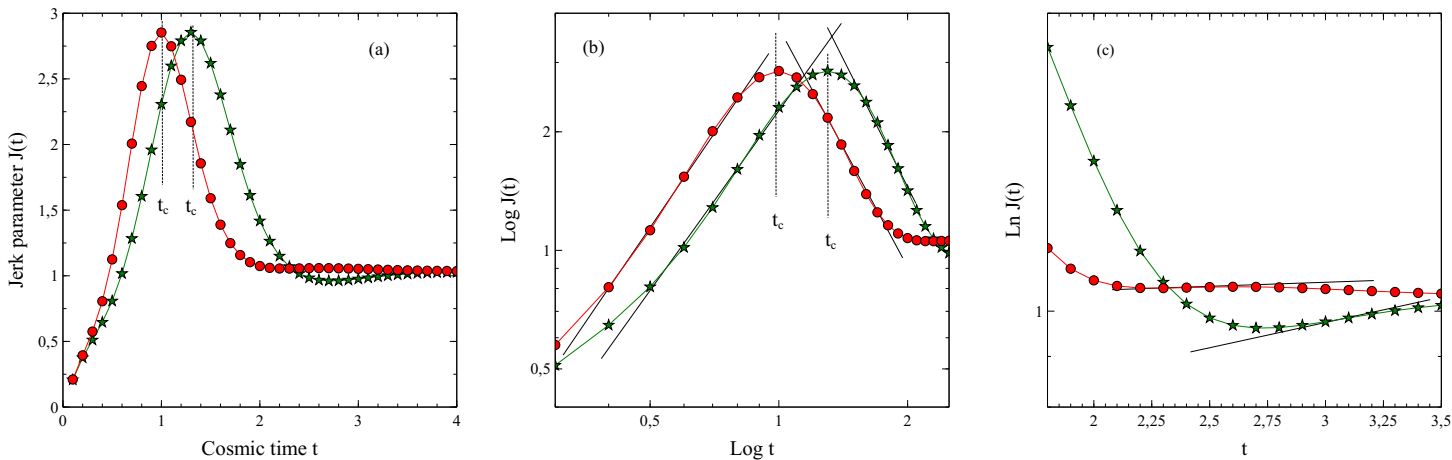

Figure 5. The jerk parameter $j(t)$ with respect to cosmic time. Here we set the parameter values $A_{1}=B_{2}=1.005$, $A_{2}=B_{1}=2.005$ and $k=0.005$ for the red circle line; $A_{1}=B_{2}=3.05, A_{2}=B_{1}=3.15$ and $k=-2.95$ for the green star line. In (b), jerk parameter $j(t)$ in Log-Log scale is provided. In (c), jerk parameter $j(t)$ in semi-log plot is shown. 
The time dependence of the jerk parameter for the present model is displayed in Fig. 5. The time-dependent behavior of the jerk parameter is shown in Fig. 5(a). However, the jerk parameter is given by log-log and semi-log scale in Figs. 5(b) and (c), respectively. Notice that the peaks appear at critical crossover times. These cusps strongly indicate a transition in the time evolution of the scale factor $a(t)$. In order to see some detailed time evolution of the jerk parameter around $t_{c}$, we give a log-log plot of the jerk parameter, as seen in Fig. 5(b). One can observe from this figure that the jerk parameter increases with a power-law exponent $j(t) \propto t^{-\gamma}$ up to critical time point $t_{c}$ and it decays with $j(t) \propto t^{-\gamma^{\prime}}$ where $\gamma$, and $\gamma^{\prime}$ denote arbitrary constant parameters. Finally, after a local minimum value, by increasing a very weak exponential with time, this parameter reaches up a constant value in the $\Lambda$ CDM model as seen Fig. 5(c).

\section{The EoS parameter $w(t)$}

Finally, we study the EoS parameter $w(t)$ as a kinematic variable. The equation of state of a perfect fluid is characterized by a dimensionless number $w$, which is equal to the ratio of its pressure $p$ to its energy density $\rho$. The equation of state may be used in FLRW equations to describe the evolution of an isotropic Universe filled with a perfect fluid. Cosmic inflation and the accelerated expansion of the Universe can be characterized by the equation of state of dark energy and it takes different values for different cosmic eras. In the simplest case, the equation of state of the cosmological constant is $w=-1$. In this case, the scale factor is given by $a(t) \sim \exp (H t)$. On the other hand, the EoS parameter can be used to distinguish the phantom and non-phantom dynamics of the Universe. The EoS parameters for the phantom and non-phantom cases are, respectively, given as $w<-1$ and $w \geq-1$. Additionally, the EoS parameter takes $w \approx 0$ in the matter dominant phase, while it takes $w=1 / 3$ in the radiation dominant phase.

The time-dependent behavior of the EoS parameter is given in Fig. 6(a). However, the EoS parameter is given by log-log and semi-log scale in Figs. 6(b) and (c), respectively. We note that log-log and semi-log figures are plotted for the absolute value of the EoS parameter after first peaks $f_{c}$ to obtain the slope of curves. Therefore in Figs. 6(b) and (c), curves are given inversely.
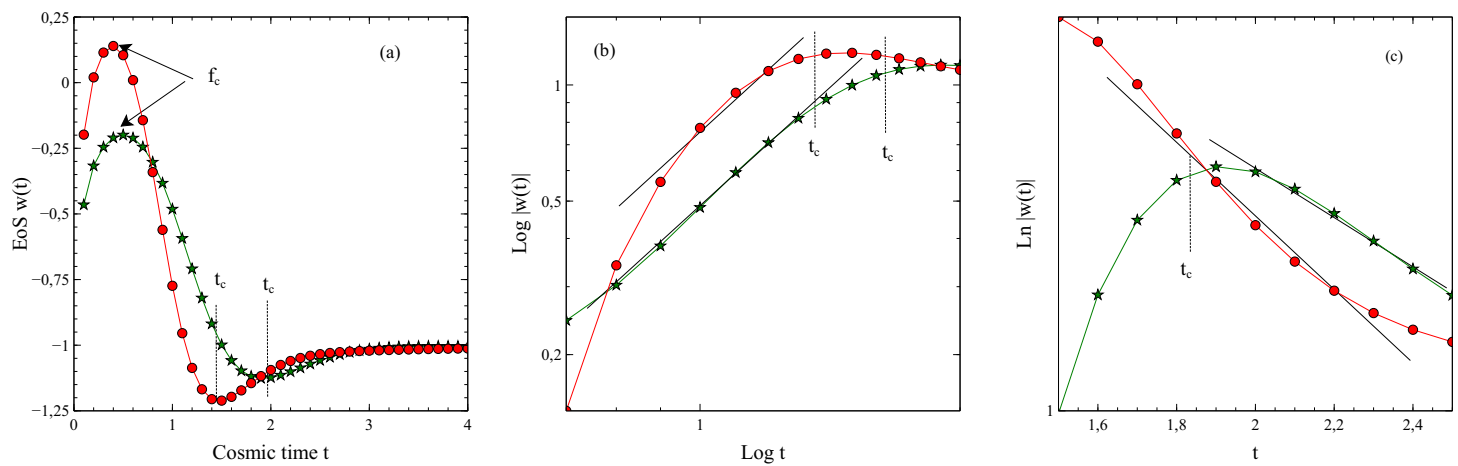

Figure 6. The EoS parameter $w(t)$ with respect to cosmic time. Here we set the parameter values $A_{1}=B_{2}=1.005$, $A_{2}=B_{1}=2.005$ and $k=0.005$ for the red circle line; $A_{1}=B_{2}=3.05, A_{2}=B_{1}=3.15$ and $k=-2.95$ for the green star line is given. In (b), EoS parameter $w(t)$ in Log-Log scale. In (c), EoS parameter $w(t)$ in semi-log plot is shown.

The EoS curves also reflect the transition in scale factor $a(t)$ around $t_{c}$ in Fig. 6(a). In addition to the deceleration parameter $q(t)$, the EoS parameter $w(t)$ also has the first initial peak which indicates a sudden acceleration in the time evolution of expansion of the Universe. EoS parameter decays with time according to a power-law $w(t) \propto t^{-\eta}$ up to critical time point $t_{c}$ as seen in Fig. 6(b). Finally, after a local minimum value, as seen in Fig. 6(c) it again exponentially increases as $w(t) \propto e^{\eta^{\prime} t}$ up to a current constant value $w=-1$ of the $\Lambda$ CDM model, as seen in Fig. 6(a) where $\eta$, and $\eta^{\prime}$ denote arbitrary constant parameters.

\section{Cosmological parameters in the Limiting Cases}

We obtained the scale factor in terms of equations of motion by solving the FLRW equation with two scalar fields, and then we plotted both the scale factor and other quantities by solving numerically. We see that scale factor can be given in the single formula by

$$
a(t)=a_{0} t^{2 / 3} e^{H_{0} t}
$$


where $a_{0}$ is the normalization constant. Now we can derive other quantities due to scale factor $a(t)$. The cosmological parameters including Hubble parameter, deceleration, jerk and EoS parameter are respectively given by

$$
\begin{aligned}
H & =\frac{\dot{a}}{a}=\frac{2}{3} t^{-1}+H_{0}, \\
q & =-\frac{\ddot{a}}{a H^{2}}=-1+\frac{6}{\left(2+3 H_{0} t\right)^{2}}, \\
j & =\frac{\dddot{a}}{a H^{3}}=1+\frac{36}{\left(2+3 H_{0} t\right)^{3}}+\frac{18}{\left(2+3 H_{0} t\right)^{2}}, \\
w & =-\frac{1}{3}-\frac{2 a \ddot{a}}{3 \dot{a}^{2}}=-1+\frac{4}{\left(2+3 H_{0} t\right)^{2}} .
\end{aligned}
$$

It is clear that one obviously obtains power-law and exponential law expansion from Eq. (28) in the limiting cases. Accordingly, for $t \rightarrow 0$, i.e. $t \leq t_{c}$, the cosmological parameters approximate to the following:

$$
a(t) \sim a_{0} t^{2 / 3}, \quad H \sim \frac{2}{3} t^{-1}, \quad q \sim 1 / 2, \quad j \sim 1 \quad \text { and } \quad w \sim 0 .
$$

Similarly, the exponential term dominates at late times, such that in the limit $t \rightarrow \infty$, i.e. $t>t_{c}$, we have

$$
a(t) \sim a_{0} e^{H_{0} t}, \quad H \rightarrow H_{0}, \quad q \rightarrow-1, \quad j \rightarrow 1, \quad \text { and } \quad w \rightarrow-1 .
$$

Notice that our results are consistent with the theoretical predictions and observational data in the limiting cases.

\section{Discussion and Conclusion}

In the introduction, we mentioned that the late time crossover between a power-law to an exponential expansion of the Universe evolution was the leading problem awaiting a recipe in physical cosmology. In this critical regime, the Universe suddenly passes from a matter-dominated period to a dark energy-dominated period. These different eras are characterized by $a(t) \propto t^{2 / 3}$ and $a(t) \propto \exp \left(H_{0} t\right)$, respectively. We know that dark energy is responsible for the late-time exponential expansion of the Universe although we have not known its physical origin. However, the existence of dark energy alone does not seem to be sufficient to explain this transition.

In this study, we propose a two-scalar fields model coupled in a Lagrangian in the framework of the FLRW metric to explain this unexpected transition-like catastrophic action. In Section II, we constructed the Lagrangian for two scalar fields which correspond to the dark matter and dark energy interacting with a potential. After we define interaction potential and discuss the stable equilibrium behavior of this potential, we solve the Lagrangian of the model and analytically obtain the equations of motion. Then, we obtained eigenvalues and eigenvectors for the model. Later, we defined the kinematic variables analytically. In Section III and IV, we numerically solved and gave our findings for all kinematic variables in Figs. 2-6. Finally, in Section V, we discuss the limit behaviors of all relevant cosmological quantities.

In the present model, two scalar fields interact with a potential which is determined by two oscillators and two antioscillators. We mainly solve the field equations in the FLRW framework for this model to obtain the time-dependent scale factor. The numerical solution of the scale factor is clearly shown in Fig. 2 that time crossover appears from a power-law $a(t) \propto t^{2 / 3}$ to an exponential expansion $a(t) \propto \exp \left(H_{0} t\right)$. This is a very interesting and important result for understanding the sudden transition between two different eras of the Universe. Furthermore, it is first time that we explicitly obtain a hybrid scale factor incorporating the power and exponential terms as $a(t) \propto t^{2 / 3} e^{H_{0} t}$. Furthermore, we numerically obtain other quantities such as Hubble $H(t)$, deceleration $q(t)$, jerk parameter $j(t)$ and $\operatorname{EoS} w(t)$ by using scale factor $a(t)$ in Figs. 3, 4, 5 and 6 , respectively. These parameters reflect different aspects of all information on the scale factor since they are obtained depending on the scale factor and/or its derivatives. Indeed, as one can notice from the relevant descriptions of figures leading to the time crossover, late time transition from power-law to the exponential one are obtained. All obtained numerical results are consistent with the observational data such as $\mathrm{BAO}$ and $\mathrm{SN} \mathrm{Ia}{ }^{1,2,54,55}$.

We mentioned in the introduction section that dark energy alone may not explain the sudden transition from power-law to the exponential expansion. Based on our finding, we conclude that two coupled scalar and interacting fields corresponding to dark matter and dark energy, respectively, offer a good mechanism to explain this late time turnover behavior. On the other hand, we notice that the chosen potential does not have a catastrophic character and does not directly lead to catastrophic dynamics. The catastrophic potentials are very special types and an extensive classification has been given by Thom in Ref. ${ }^{22}$. Here, to find an exact solution, we linearized the potential in Eq. (7) by Taylor series expansion. One can see from Eq. (11) that the potential, including higher-order terms, has polynomial characters. Thus, our interaction potential is approximately fitting to any catastrophic potential. It serves to establish a relation between the sudden transition and the interaction potential. 
In summary, we consider the interaction of two scalar fields corresponding to dark matter and dark energy in the FRLW framework. We show by using this interacting model that the late time observational crossover of the Universe can be explained. We present a hybrid scale factor formula which is directly obtained from our model itself. The method we use here can be generalized to the many scalar fields interacting linearly or non-linearly. In this sense, it can be regarded that this work serves as a supportive practical application of the study about metric dependent modeling of Chaotic Universe Theory proposed in Ref. ${ }^{28}$. In the next step, we will discuss how to scale the results obtained by trying to determine the parameters we use in the model using observational BAO and SN Ia data. Additionally, we will study the generalization of the method presented here to Chaotic Universe Theory to obtain the metric dependent solutions.

\section{References}

1. Riess, A. G. et al. Observational evidence from supernovae for an accelerating universe and a cosmological constant. The Astron. J. 116, 1009-1038, DOI: 10.1086/300499 (1998). astro-ph/9805201.

2. Perlmutter, S. et al. Measurements of $\omega$ and $\lambda$ from 42 high redshift supernovae. The Astrophys. J. 517, 565-586, DOI: 10.1086/307221 (1999).

3. Linde, A. D. A new inflationary universe scenario: A possible solution of the horizon, flatness, homogeneity, isotropy and primordial monopole problems. Phys. Lett. B 108, 389 - 393, DOI: 10.1016/0370-2693(82)91219-9 (1982).

4. Weinberg, S. Cosmology (Oxford University Press, USA, 2008).

5. Barreiro, T., Copeland, E. J. \& Nunes, N. J. Quintessence arising from exponential potentials. Phys. Rev. D 61, 127301, DOI: 10.1103/PhysRevD.61.127301 (2000). astro-ph/9910214.

6. Caldwell, R. R. A phantom menace? cosmological consequences of a dark energy component with super-negative equation of state. Phys. Lett. B 545, 23 - 29, DOI: 10.1016/S0370-2693(02)02589-3 (2002). astro-ph/9908168.

7. Armendariz-Picon, C., Mukhanov, V. \& Steinhardt, P. J. Essentials of k-essence. Phys. Rev. D 63, 103510, DOI: 10.1103/ PhysRevD.63.103510 (2001). astro-ph/0006373.

8. Bagla, J. S., Jassal, H. K. \& Padmanabhan, T. Cosmology with tachyon field as dark energy. Phys. Rev. D 67, 063504, DOI: 10.1103/PhysRevD.67.063504 (2003). astro-ph/0212198.

9. Bento, M. C., Bertolami, O. \& Sen, A. A. Generalized chaplygin gas, accelerated expansion, and dark-energy-matter unification. Phys. Rev. D 66, 043507, DOI: 10.1103/PhysRevD.66.043507 (2002). gr-qc/0202064.

10. Li, M. A model of holographic dark energy. Phys. Lett. B 603, 1 - 5, DOI: 10.1016/j.physletb.2004.10.014 (2004). hep-th/0403127.

11. Capozziello, S. \& Roshan, M. Exact cosmological solutions from hojman conservation quantities. Phys. Lett. B 726, 471-480, DOI: 10.1016/j.physletb.2013.08.047 (2013). 1308.3910.

12. Belinchón, J. A., Harko, T. \& Mak, M. K. Exact scalar-tensor cosmological models. Int. J. Mod. Phys. D 26, 1750073, DOI: $10.1142 / \mathrm{S} 0218271817500730$ (2017). 1612.05446.

13. Tajahmad, B. Studying the intervention of an unusual term in $f(t)$ gravity via the noether symmetry approach. Eur. Phys. J. C 77, 510, DOI: 10.1140/epjc/s10052-017-5050-z (2017). 1701.01620.

14. Sharif, M. \& Nawazish, I. Cosmological analysis of scalar field models in $f(r, t)$ gravity. Eur. Phys. J. C 77, 198, DOI: 10.1140/epjc/s10052-017-4773-1 (2017). 1703.06763.

15. Paliathanasis, A. \& Leon, G. Analytic solutions in einstein-aether scalar field cosmology. Eur. Phys. J. C 80, 355, DOI: 10.1140/epjc/s10052-020-7924-8 (2020). 2003.03903.

16. Paliathanasis, A. \& Tsamparlis, M. Two scalar field cosmology: Conservation laws and exact solutions. Phys. Rev. D 90, 043529, DOI: 10.1103/PhysRevD.90.043529 (2014). 1408.1798.

17. Kucukakca, Y. \& Akbarieh, A. R. Noether symmetries of einstein-aether scalar field cosmology. Eur. Phys. J. C 80, 1019, DOI: 10.1140/epjc/s10052-020-08583-7 (2020).

18. Nojiri, S., Odintsov, S. \& Oikonomou, V. Modified gravity theories on a nutshell: Inflation, bounce and late-time evolution. Phys. Reports 692, 1 - 104, DOI: https://doi.org/10.1016/j.physrep.2017.06.001 (2017). Modified Gravity Theories on a Nutshell: Inflation, Bounce and Late-time Evolution.

19. Nojiri, S. \& Odintsov, S. Unifying phantom inflation with late-time acceleration: scalar phantomnon-phantom transition model and generalized holographic dark energy. Gen. Relativ. Gravit. 38, 1572-9532, DOI: https://doi.org/10.1007/ s10714-006-0301-6 (2006). 
20. Oz, I., Kucukakca, Y. \& N.Unal. Anisotropic solution in phantom cosmology via noether symmetry approach1. Can. J. Phys. 96, 677-680, DOI: 10.1139/cjp-2017-0765 (2018).

21. Bahamonde, S. et al. Dynamical systems applied to cosmology: Dark energy and modified gravity. Phys. Reports 775-777, 1 - 122, DOI: https://doi.org/10.1016/j.physrep.2018.09.001 (2018).

22. Thom, R. Structural Stability and Morphogenesis: An Outline of a General Theory of Models. Advanced book classics (CRC Press, Taylor \& Francis GroupPerseus Books, 1989).

23. Caldwell, R. R., Dave, R. \& Steinhardt, P. J. Cosmological imprint of an energy component with general equation of state. Phys. Rev. Lett. 80, 1582-1585, DOI: 10.1103/PhysRevLett.80.1582 (1998). astro-ph/9708069.

24. Liddle, A. R. \& Scherrer, R. J. Classification of scalar field potentials with cosmological scaling solutions. Phys. Rev. D 59, 023509, DOI: 10.1103/PhysRevD.59.023509 (1998). astro-ph/9809272.

25. Peebles, P. \& Ratra, B. The cosmological constant and dark energy. Rev. Mod. Phys. 75, 559-606, DOI: 10.1103/ RevModPhys.75.559 (2003). astro-ph/0207347.

26. Wetterich, C. Cosmology and the fate of dilatation symmetry. Nucl. Phys. B 302, 668 - 696, DOI: 10.1016/0550-3213(88) 90193-9 (1988). 1711.03844.

27. Barrow, J. D. Quiescent cosmology. Nature 272, 211-215, DOI: 10.1038/272211a0 (1978).

28. Aydiner, E. Chaotic universe model. Sci. Reports 8, 721, DOI: 10.1038/s41598-017-18681-4 (2018).

29. Brax, P. \& Martin, J. The supergravity quintessence model coupled to the minimal supersymmetric standard model. J. Cosmol. Astropart. Phys. 11, 008, DOI: 10.1088/1475-7516/2006/11/008 (2006). astro-ph/0606306.

30. Bolotin, Y. L., Kostenko, A., Lemets, O. \& Yerokhin, D. Cosmological evolution with interaction between dark energy and dark matter. Int. J. Mod. Phys. D 24, 1530007, DOI: 10.1142/S0218271815300074 (2015). 1310.0085.

31. Zimdahl, W. \& Pavon, D. Statefinder parameters for interacting dark energy. Gen. Relativ. Gravit. 36, 14831491, DOI: 10.1023/B:GERG.0000022584.54115.9e (2004). gr-qc/0311067.

32. Amendola, L., Campos, G. C. \& Rosenfeld, R. Consequences of dark matter-dark energy interaction on cosmological parameters derived from type ia supernova data. Phys. Rev. D 75, 083506, DOI: 10.1103/PhysRevD.75.083506 (2007). astro-ph/0610806.

33. Wang, B., Abdalla, E., Atrio-Barandela, F. \& Pavon, D. Dark matter and dark energy interactions: theoretical challenges, cosmological implications and observational signatures. Reports on Prog. Phys. 79, 096901, DOI: 10.1088/0034-4885/ 79/9/096901 (2016). 1603.08299.

34. Böhmer, C. G., Tamanini, N. \& Wright, M. Interacting quintessence from a variational approach. i. algebraic couplings. Phys. Rev. D 91, 123002, DOI: 10.1103/PhysRevD.91.123002 (2015). 1501.06540.

35. Böhmer, C. G., Tamanini, N. \& Wright, M. Interacting quintessence from a variational approach. ii. derivative couplings. Phys. Rev. D 91, 123003, DOI: 10.1103/PhysRevD.91.123003 (2015). 1502.04030.

36. He, J.-H. \& Wang, B. Effects of the interaction between dark energy and dark matter on cosmological parameters. J. Cosmol. Astropart. Phys. 06, 010, DOI: 10.1088/1475-7516/2008/06/010 (2008). 70801.4233.

37. Cai, R.-G., Tamanini, N. \& Yang, T. Reconstructing the dark sector interaction with LISA. J. Cosmol. Astropart. Phys. 05, 031, DOI: 10.1088/1475-7516/2017/05/031 (2017). 1703.07323.

38. Yang, W., Banerjee, N., Paliathanasis, A. \& Pan, S. Reconstructing the dark matter and dark energy interaction scenarios from observations. Phys. Dark Universe 26, 100383, DOI: 10.1016/j.dark.2019.100383 (2019). 1812.06854.

39. Amendola, L. Coupled quintessence. Phys. Rev. D 62, 043511, DOI: 10.1103/PhysRevD.62.043511 (2000). astro-ph/ 9908023.

40. Tocchini-Valentini, D. \& Amendola, L. Stationary dark energy with a baryon-dominated era: Solving the coincidence problem with a linear coupling. Phys. Rev. D 65, 063508, DOI: 10.1103/PhysRevD.65.063508 (2002). astro-ph/0108143.

41. Amendola, L. \& Quercellini, C. Tracking and coupled dark energy as seen by the wilkinson microwave anisotropy probe. Phys. Rev. D 68, 023514, DOI: 10.1103/PhysRevD.68.023514 (2003). astro-ph/0303228.

42. del Campo, S., Herrera, R. \& Pavon, D. Toward a solution of the coincidence problem. Phys. Rev. D 78, 021302, DOI: 10.1103/PhysRevD.78.021302 (2008). 0806.2116.

43. del Campo, S., Herrera, R. \& Pavon, D. Interacting models may be key to solve the cosmic coincidence problem. J. Cosmol. Astropart. Phys. 020, DOI: 10.1088/1475-7516/2009/01/020 (2009). 0812.2210. 
44. Wei, H. \& Zhang, S. N. Observational h(z) data and cosmological models. Phys. Lett. B 644, 7 - 15, DOI: 10.1016/j. physletb.2006.11.027 (2007). astro-ph/0609597.

45. del Campo, S., Herrera, R. \& Pavón, D. Interaction in the dark sector. Phys. Rev. D 91, 123539, DOI: 10.1103/PhysRevD. 91.123539 (2015). 1507.00187.

46. Chimento, L. P. Linear and nonlinear interactions in the dark sector. Phys. Rev. D 81, 043525, DOI: 10.1103/PhysRevD. 81.043525 (2010). 0911.5687.

47. Sanchez, G. \& Ivan, E. Dark matter interacts with variable vacuum energy. Gen. Rel. Grav. 46, 1769, DOI: 10.1007/ s10714-014-1769-0 (2014). 1405.1291.

48. Verma, M. M. Dark energy as a manifestation of the non-constant cosmological constant. Astrophys. Space Sci 330, 101-105, DOI: 10.1007/s10509-010-0347-5 (2010).

49. Shahalam, M., Pathak, S. D., Verma, M. M., Khlopov, M. Y. \& Myrzakulov, R. Dynamics of interacting quintessence. Eur. Phys. J. C 75, 395, DOI: 10.1140/epjc/s10052-015-3608-1 (2015). 1503.08712.

50. Cruz, M. \& Lepe, S. Holographic approach for dark energy-dark matter interaction in curved FLRW spacetime. Class. Quantum Gravity 35, 155013, DOI: 10.1088/1361-6382/aacd9e (2018).

51. Cruz, M., Lepe, S. \& Morales-Navarrete, G. Qualitative description of the universe in the interacting fluids scheme. Nucl. Phys. B 943, 114623, DOI: https://doi.org/10.1016/j.nuclphysb.2019.114623 (2019).

52. Saleem, R. \& Imtiaz, M. J. Dynamical study of interacting ricci dark energy model using chevallier-polarsky-lindertype parametrization. Class. Quantum Gravity 37, 065018, DOI: 10.1088/1361-6382/ab6fof (2020).

53. Dereli, T. \& Tucker, R. W. Signature dynamics in general relativity. Class. Quantum Gravity 10, 365-374, DOI: 10.1088/ 0264-9381/10/2/018 (1993).

54. Clocchiatti, A. et al. Hubble space telescopeand ground-based observations of type ia supernovae at redshift 0.5: Cosmological implications. The Astrophys. J. 642, 1-21, DOI: 10.1086/498491 (2006).

55. Planck Collaboration. Planck 2018 results - vi. cosmological parameters. A@AND@A 641, A6, DOI: 10.1051/0004-6361/ 201833910 (2020).

\section{Acknowledgements}

The author Isil Basaran-Öz grateful to Istanbul University for hospitality and grant for the Post-Doctoral Researcher Position at Istanbul University. This work was supported by Istanbul University Post-Doctoral Research Project: MAB-2019-33386 which is titled "The metric dependent investigation of Chaotic Universe Theory".

\section{Author contributions statement}

E. A. and T. D. developed the theory. All co-authors performed to obtain the analytical solutions. E. A. and I. B.-O. obtained the numerical results and plotted figures. E. A and T. D. interpreted the results. E. A. wrote the manuscript. All co-authors revised the manuscript.

\section{Corresponding author}

Correspondence to Ekrem Aydiner

\section{Additional information}

The author declares that there is no competing interests. 